\title{
Clinical impact and outcome of clostridium non-difficile infection in critically ill patients
}

\author{
K Rutter", S Walther, T Horvatits, A Drolz, K Roedl, C Bopp, R Wüstenberg, S Kluge, V Fuhrmann \\ From ESICM LIVES 2015 \\ Berlin, Germany. 3-7 October 2015
}

\section{Introduction}

Clostridium species are gram-positive, anaerobic, sporeforming bacteria and some species have pathogenic nature. There is limited data regarding Clostridium non-difficile infection in critically ill patients available. Symptoms of infection are often non-specific, which leads to delayed diagnosis and therapy initiation in these patients.

\section{Objectives}

Aim of this study was to evaluate patients with positive microbiological cultures of Clostridium non-difficile infection with the need of critical care medicine.

\section{Methods}

Patients with microbiological result of Clostridium nondifficile infection admitted to the intensive care unit (ICU) were included in this study. Patient's characteristics including admission diagnosis, severity of illness (SOFA-score), therapeutic procedures and outcome were recorded.

\section{Results}

A total of 47 critically ill patients (32 men, mean age $66 \pm$ 9 years, mean SOFA-score on admission $9 \pm 3$ ) with Clostridium non-difficile infection were included in this study. The most common pathogens were Clostridium innocum $(\mathrm{n}=23)$, Clostridium perfringens $(\mathrm{n}=11)$, Clostridium tertium $(n=6)$ and others $(n=7)$. Pathogens were detected in $62 \%$ intra-abdominal, in $29 \%$ in blood cultures and $8 \%$ of patients had soft tissue infection. Intra-abdominal infections $(71 \%)$ were the most common source of infection.

Admission diagnoses were septic shock (54\%), surgical treatment (44\%) and others (2\%). Highest incidence of septic shock was seen in patients with Clostridium innocum infection, $(\mathrm{p}<0.05)$. Preexisting, mostly abdominal (90\%) malignancy was seen in $46 \%$ of these patients. Invasive ventilation was needed in $50 \%$, vasopressor therapy in $71 \%$ and renal replacement therapy in $38 \%$. The overall ICU mortality was $45 \%$. Highest ICU mortality rate was found in patients with Clostridium innocum infection (45\%), followed by Clostridium perfringens (23\%) and Clostridium tertium (17\%). Patients with septic shock showed significantly higher mortality rates $(p<0.05)$. By means of source of infection highest mortality rate was found in abdominal infection $(65 \%)$ followed by bacteremia $(43 \%)$.

\section{Conclusions}

Clostridium non-difficile infection in critically ill patients is associated with high mortality and organ failure. Worst outcome was observed in patients with septic shock.

Published: 1 October 2015

doi:10.1186/2197-425X-3-S1-A117

Cite this article as: Rutter et al:: Clinical impact and outcome of clostridium non-difficile infection in critically ill patients. Intensive Care Medicine Experimental 2015 3(Suppl 1):A117. 